This is the peer reviewed version of the following article: [Transfusion 59(11):3405-3412(2019)], which has been published in final form at [https://doi.org/10.1111/trf.15527. ]

This article may be used for non-commercial purposes in accordance with Wiley Terms and Conditions for Use of Self-Archived Versions.

Transfusion 59(11) :3405-3412(2019) 


\title{
Investigation of factors associated with allergic transfusion reaction due to platelet transfusion and the efficacy of platelets resuspended in BRS-A in adult patients
}

Manjiro Yamanaka ${ }^{1}$, Ryu Yanagisawa ${ }^{1,2}$, Shunsuke Kojima ${ }^{1,2}$, Hideyuki Nakazawa ${ }^{3}$, and Shigetaka Shimodaira ${ }^{4}$

1 Division of Blood Transfusion, Shinshu University Hospital, Matsumoto, Japan

2 Center for Advanced Cell Therapy, Shinshu University Hospital, Matsumoto, Japan

3 Division of Hematology, Department of Internal Medicine, Shinshu University School of Medicine, Matsumoto, Japan

4 Department of Regenerative Medicine, Kanazawa Medical University, Uchinada-Cho, Kahoku-Gun, Japan

\section{CONFLICT OF INTEREST}

The authors have no potential conflicts of interest to declare.

CORRESPONDING AUTHOR: Ryu Yanagisawa, $\mathrm{MD}, \mathrm{PhD}$

Division of Blood Transfusion, Shinshu University Hospital

3-1-1, Asahi, Matsumoto 390-8621, Japan

Tel: +81-263-373240; Fax: +81-263-373027

E-mail: ryu@shinshu-u.ac.jp

RUNNING TITLE: Factors associated with ATR and the use of PC resuspended in BRS-A

\begin{abstract}
BACKGROUND: Although allergic transfusion reactions (ATRs) resulting from platelet concentrate (PC) are a common adverse reaction, the mechanism underlying ATRs has not been fully elucidated. Plasma-replaced PC suspended in bicarbonate Ringer's solution and anticoagulant citrate dextrose solution A (RPC-B) is effective for preventing ATRs in children in Japan; however, there is not enough evidence in adult populations.

STUDY DESIGN AND METHODS: We conducted a retrospective analysis focused on factors associated with ATRs developing from PC transfusions in adult patients in a single institution between 2015 and 2018. The clinical efficacy of RPC-B for adult patients was also analysed.

RESULTS: In total, 4,677 untreated regular PC products in plasma were transfused into 914 patients. ATRs developed in 65 patients $(7.1 \%)$ treated with 92 PC products $(2.0 \%)$. Multivariate analysis revealed that patients who were elderly, diagnosed with a non-haematological disease, and who
\end{abstract}


received a transfusion of fresh-frozen plasma and red blood cell concentrate products together with PC products had lower frequencies of ATRs. Although 41 patients received 490 RPC-B transfusions, six ATRs (1.2\%) were confirmed in five patients (12.5\%). The ATR frequency was not significantly lower in the analysis of all patients; however, ATRs in patients with haematological diseases were lower in terms of both the patient and product numbers. Corrected count increments $(24 \mathrm{~h})$ were also within an acceptable range in patients with haematological diseases.

CONCLUSION: Several patient-specific factors may be associated with the development of ATRs from PC transfusion. Because RPC-B appears to efficiently prevent ATRs, even in adult patients, safe and efficient transfusions may be performed by using RPC-B preferentially depending on the patient's risk factors.

KEY WORDS: adverse transfusion reaction, apheresis platelet, bicarbonate Ringer's solution, platelet additive solution, resuspended platelet concentrate

\section{Introduction}

Allergic transfusion reactions (ATRs) resulting from the use of platelet concentrate (PC) are the most common transfusion-related adverse reaction. ${ }^{1,2}$ Serious symptoms, such as anaphylaxis, are rare; most ATRs that develop are mild. ${ }^{3,4}$ Although specific antibodies were identified as causal factors in the development of severe ATRs, the full mechanism of the development of ATRs, including the mild type, has not been fully elucidated. ${ }^{4,5}$ Factors related to the products included in the plasma of the blood products are considered to be important factors in the development of ATRs. ${ }^{6-10}$ However, other patient-related factors associated with ATRs may exist $t^{4-7}$ because a specific patient could develop multiple ATRs in the medical setting. ATRs are thought to be caused by not only a specific factor but also multiple complex factors. ${ }^{4}$ Therefore, it is necessary to investigate many factors associated with ATR development, such as donor-, product-, and recipient-related factors. ${ }^{2,4-8}$ However, it is usually difficult to investigate all of these factors in a single study. Although previous studies have revealed several causal factors of ATR, ${ }^{4-6,11}$ with regard to recipient factors, the active cooperation of medical staff who use blood products in the medical setting is necessary. Additionally, detailed clinical information of the patients is required, including information from the patients who did not develop ATRs for the purpose of comparison. Therefore, investigating recipient factors associated with ATRs is not always easy, and the evidence remains insufficient.

Transfusion premedication is used to prevent ATRs, but clear protective evidence for this approach has not yet been demonstrated. ${ }^{12-14}$ On the other hand, it has been reported that ATRs could be prevented effectively by introducing plasma-removed PC products, such as washed PC products. ${ }^{6,15}$ 
Because commercial platelet additive solution (PAS) is not available in Japan, PAS should be prepared before adjusting plasma-removed PC products. Usually, we mix several kinds of infusion products. Recently, a mixture of bicarbonate Ringer's solution and anticoagulant citrate dextrose solution A (BRS-A) was reported to be a relatively easily prepared PAS with potential to maintain platelet qualities for a longer period. ${ }^{16,17}$ Although the Japanese Red Cross Blood Center started to provide automatically adjusted washed PC with BRS-A, ${ }^{18,19}$ a limited quantity of this kind of washed PC is available each day. Furthermore, prior planning is necessary for the use of washed PC with BRS-A. Therefore, in institutions in which the flexible management of blood products is required, washed PC products should be prepared manually in each institution. Recently, manually removed plasma and PC products resuspended in BRS-A (RPC-B) have also been reported as safe and effective transfusion products in paediatric populations; ${ }^{15,20}$ however, evidence in adult patients does not exist.

Therefore, we conducted a detailed retrospective investigation focused specifically on adult recipients' factors regarding ATR development due to PC transfusion in a single institution. Furthermore, the clinical safety and efficacy of RPC-B for the ATR-developing adult patients in this study were also analysed.

\section{Materials and Methods}

\section{Patient selection and transfused PC products}

This retrospective study was conducted with adult patients $>20$ years old who received transfusions with untreated regular PC in plasma (P-PC) at Shinshu University Hospital between October 2015 and September 2018. All P-PC was prepared by apheresis of blood derived from a single donor at the Japanese Red Cross Blood Center. For all PC products, pre-storage leukocyte reduction and diversion of the first aliquot of blood were performed. ${ }^{15,20}$ All P-PCs were supplied after the performance of a nucleic acid amplification test for HBV, HCV, and HIV; thereafter, the components were exposed to irradiation from 15 to $50 \mathrm{~Gy}$ and were used within 4 days of apheresis..$^{15,20,21} \mathrm{We}$ prepared a simplified RPC-B for the patients who developed ATR if the patients satisfied the following criteria. ${ }^{15,20,21} 1$ ) Cases in which side effects that could not be prevented with the pre-administration of various drugs were observed two or more times. 2) Cases in which serious side effects, such as anaphylactic shock, were observed once. BRS-A and RPC-Bs were prepared at the Shinshu University Hospital according to the Japan Society of Transfusion Medicine and Cell Therapy guidelines and previous reports. ${ }^{15,20,21}$ Pretransfusion medication, such as acetaminophen or an antihistamine, was not provided routinely. The Institutional Review Board of the institution approved this study (approval number: 4366). Written informed consent was provided by the patients before the transfusion.

\section{Evaluation of ATRs and transfusion efficacy}

Reactions to the transfused PC products were recorded during the transfusion and follow-up periods, 
and data regarding the type of adverse reaction (ATR or a non-allergic event including transfusionassociated infections) were also recorded. All transfusion reactions recognized by physicians and/or nurses were reported to the Division of Blood Transfusion in Shinshu University Hospital during the study period. The types of ATRs assessed have been described elsewhere. ${ }^{15,20}$ ATRs were defined as the presence of at least one of the following symptoms during transfusion or within 4 hours of transfusion: maculopapular rash with/without pruritus; urticaria; pruritus; generalized flushing; localized angioedema; oedema of the lips, tongue, or uvula; erythema and oedema of the periorbital area; conjunctival oedema; respiratory distress; bronchospasm; and hypotension. We calculated the posttransfusion corrected count increment $(\mathrm{CCI})(24 \mathrm{hr})\left(\times 10^{10} / \mathrm{L}\right)$ to evaluate the transfusion effect based on a previously described method. ${ }^{15,20,21} \mathrm{CCI}$ was evaluated in each patient within 6 months of the initial PC transfusion for a maximum of 10 PC products. Transfusions in which aggravation of the primary disease resulted in refractoriness to transfusion, fever, infection, immunological responses such as graft-versus-host disease, disseminated intravascular coagulation, splenomegaly, or bleeding/bleeding tendency were excluded from the CCI analysis. ${ }^{15}$

\section{Statistical methods}

We compared the differences in recipient factors expressed as a concrete number (e.g., body height and body weight) using the Mann-Whitney U test and Fisher's exact test between patients with and without ATRs. Other potential recipient risk factors were evaluated using Fisher's exact test and logistic regression analysis. Potential factors with at least borderline significance $(\mathrm{P}<0.15)$ identified using univariate analysis were included in multivariate analyses. The frequency of ATRs associated with P-PC or RPC-B transfusions was analysed with Fisher's exact test. The posttransfusion CCIs (24 hr) of P-PC and RPC-B were compared using the Mann-Whitney U test. Statistical analyses were performed using EZR (Saitama Medical Center, Jichi Medical University, Saitama, Japan) ${ }^{22}$ and SPSS Statistics 23 (IBM Corporation, Armonk, NY, USA). Statistical significance was defined as $\mathrm{P}<0.05$.

\section{Results}

\section{Frequency of ATRs and the associated recipient factors}

During the study period, a total of 4,677 P-PC products were transfused into 914 patients. ATRs were developed in 65 patients (7.1\%) treated with 92 P-PC products (2.0\%). In the analysis of the patients' backgrounds, there were no differences in physical characteristics of the patients (height, weight, body surface area, and body mass index) with and without ATRs. However, patients who developed ATRs were younger than the patients who did not. The risk ratio of a younger age to older age shown as the ratio of the ATR incidence in patients under and over the median age was 6.16 (95\% confidence interval (CI) 3.18-11.94, $\mathrm{P}<0.001$ ) (Table 1). The univariate analysis of 11 recipient factors included 
the candidate, difference in age, difference in underlying disease, history of allergies (drugs and foods), transfused period, and the use of other transfused blood products during this study period (Table 2A). Multivariate analysis included the difference in age (odds ratio 0.470 ( $95 \%$ CI $0.313-0.706$ ) $\mathrm{p}<0.001$ ), underlying disease (odds ratio $0.429(95 \%$ CI $0.315-0.584) \mathrm{p}<0.001)$, and the use of other transfused blood products (odds ratio 0.457 (95\% CI 0.353-0.592) $\mathrm{p}<0.001$ ), which were extracted as ATRassociated factors (Table 2B).

We show the detailed odds ratios in the breakdown of these items and the corresponding probabilities in Table 3. Regarding age, patients $>60$ years were at a significantly lower risk of having an ATR than patients $<40$ years (odds ratio 0.247 (95\% CI 0.104-0.588) $\mathrm{p}=0.002$ ). Haematological disease carried a higher risk of an ATR developing than all of the other diseases (haematological disease vs emergent disease, odds ratio 0.098 (95\% CI 0.019-0.496) $\mathrm{p}=0.005$; haematological disease vs surgical disease, odds ratio 0.125 (95\% CI 0.028-0.559) $\mathrm{p}=0.006$; haematological disease vs other disease, odds ratio 0.108 (95\% CI 0.049-0.240) $\mathrm{p}<0.001)$. However, there were no other significant differences when comparing the other diseases. Regarding the use of other transfused blood products, the use of red blood cell (RBC) and fresh-frozen plasma (FFP) products carried a lower risk of an ATR developing than the transfusion of only PC products or $\mathrm{RBC}$ and $\mathrm{PC}$ products (none vs $\mathrm{RBC}$ and FFP, odds ratio 0.155 (95\% CI 0.047-0.514) $\mathrm{p}=0.002 ; \mathrm{RBC}$ vs $\mathrm{RBC}$ and FFP, odds ratio 0.253 (95\% CI 0.077-0.833) $\mathrm{p}=0.024)$. Because there were no cases of an ATR with the use of FFP product combinations, we were not able to calculate the odds ratio for this situation.

\section{The efficacy of introduction of RPC-B in patients with ATRs}

Of the patients who developed ATRs, 40 met the criteria for the introduction of RPC-B, and 490 RPCB products were prepared and transfused. Even after the introduction of RPC-B, six ATRs (1.2\%) were confirmed in five patients (12.5\%) who underwent transfusion with RPC-B. The frequency of ATRs was not significantly reduced in the analysis of all of the patients after the introduction of RPCB based on the calculations per patient and per product. However, when we performed the same analysis according to the patient's underlying disease, a reduction in ATRs was confirmed in the haematological disease group in terms of both per patient $(p=0.019)$ and per product $(p=0.006)$ numbers. The reduction in ATRs per blood product was more remarkable when we made comparisons within the limited individuals who developed ATRs and in whom RPC-B products were switched during the study period. Only patients with haematological disease met these criteria. The frequency of ATRs per product was reduced from 9.3\% for P-PC products to 1.1\% for RPC-B products in patients with haematological disease $(\mathrm{p}<0.001)$ (Table 4).

Although there 1,020 data points as candidates for the CCI calculation and analysis, 539 data points were eliminated because of discrepant conditions regarding the transfused period or the number of transfused products in each patient. Furthermore, 184 data points were eliminated because of 
discrepancies in the clinical condition of the patients. Finally, 239 data points were available for the CCI analysis in this study. The CCI was not significantly different between P-PC and RPC-B when we analysed them for each underlying disease (i.e., haematological diseases only or other diseases). However, the CCI of RPC-B was lower than that of P-PC when we compared it with the data of the total patient sample (Table 4). No other specific adverse events associated with RPC-B were observed during the study period.

\section{Discussion}

In this study of adult patients who developed ATRs, it was shown that the frequency of ATRs as a result of using PC products was lower in the patients who were elderly, who were diagnosed with a non-haematological disease, and who received a transfusion with RBC and FFP products during the study period. Patients with haematological diseases usually require a large number of transfusion products for a long period of time. This factor may be one of the reasons for the differences in the ATR frequency according to the different diagnoses. ${ }^{3}$ Although we included both of these factors, i.e., the number of transfused PC products and transfusion periods in our analysis, neither factor was statistically significant. Additionally, a previous report showed that the number of transfusions did not always influence the frequency of ATRs. ${ }^{23,24}$ Therefore, the higher frequency of ATRs in patients with haematological disease may be associated with disease-specific characteristics instead of the transfusion number and transfusion period. Interestingly, a similar bias in terms of disease diagnosis of haematological and/or malignant diseases was also observed in the paediatric population. ${ }^{25}$ Although the reason for the higher frequency of ATRs in adults with haematological diseases has not been revealed, haematological malignant diseases are known to be a cause of eosinophilia. ${ }^{26}$ Eosinophilia is considered to be associated with the spontaneous secretion of IL-5 from tumour cells or overproduction of T cells with IL-2 during chemotherapy. ${ }^{26}$ Because eosinophilia is an important finding in allergic disease, a haematological disease-specific background may be associated with the higher frequency of ATRs in patients with haematological disease. On the other hand, it has also been speculated that a high frequency of ATRs may be attributed to Th1/Th2 cytokine profile imbalances associated with haematological and/or malignant disease. ${ }^{25}$ While these disease-specific considerations may improve the understanding of ATRs, further investigation will be necessary because there is insufficient evidence to support this hypothesis.

Regarding the transfusion patient's age, children had a higher frequency of ATRs resulting from PC products than adults in the previous study. ${ }^{2,27}$ On the other hand, it was shown that the occurrence of transfusion complications was higher in older children than in younger children. ${ }^{25,28}$ Although our study did not include a paediatric population, the frequency of ATR development after PC transfusion was higher in the younger adult population. Therefore, the actual immune response associated with 
ATRs may develop at a higher frequency in the generation between older children and younger adults. However, the hypothesis of the biased frequency of ATR development according to age distribution was not always confirmed in other reports. Although Oakley et al. also analysed the distribution of age and frequency of ATR occurrence, their results did not seem to match ours. ${ }^{2}$ Because ATRs are not caused by a single causal factor, multiple factors may combine to affect the occurrence of ATR. Because racial differences among patients has also been shown to influence the frequency of the development of ATRs, ${ }^{28}$ further analysis is necessary to determine whether our results are Japanesespecific or generalizable to other races in other regions of the world.

In the analysis of other transfused products, the use of RBC and FFP products carried a lower risk for developing an ATR. Because the combination of RBC and FFP products carried a lower risk than the use of no other products or the use of RBC products, the use of FFP products seems to be important for reducing the risk of ATRs. Compared to red blood cell products that contain fewer plasma components, PC and FFP products were usually associated with a higher frequency of ATR occurrence. ${ }^{2,29}$ Because the presence of plasma is considered to be one of the important conditions for the occurrence of ATRs, ${ }^{6,7,10}$ combining FFP and PC products was expected to increase the occurrence of ATRs. However, the actual result was the opposite. Although FFP was one of the products associated with the highest rate of ATR development, the frequency of ATR development associated with FFP seemed to be lower than that associated with PC products. ${ }^{1,2,10,29}$ Therefore, it is speculated that the factors in plasma that are associated with ATR development could be modified or reduced by freezing and thawing the plasma. On the other hand, platelets are reported to release several cytokines that are involved with ATR during storage. ${ }^{30,31}$ Fewer cytokine-like substances are released during storage in FFP products because FFP is frozen immediately after drawing blood; this may also be associated with a lower frequency of ATRs in FFP products. Although it is not known why transfusion with the addition of FFP reduced the frequency of ATRs associated with PC products, desensitization and reductions in ATR development after repeated PC transfusions have been reported. ${ }^{11,32}$ Therefore, the combined repeated transfusion of FFP products, which already have reduced factors associated with ATR development, might result in desensitization in terms of ATRs, and as a result, there was an advantage evidenced by the lower frequency of ATRs. However, further basic investigations will be necessary to determine whether this speculation is correct.

In this study, we confirmed the ATR preventive efficacy of the use of RPC-B for adult patients with haematological diseases. However, the results of the other patients without haematological diseases did not reach statistical significance, which may be attributed to the small number of patients who received RPC-B transfusion in the other patient groups. Although the presence of a haematological disease is one of the important recipient factors associated with the occurrence of ATRs, it was 
confirmed again that the existence of plasma in PC products was strongly necessary for the onset of the ATRs. However, the CCI associated with the transfusion of RPC-B was lower in the data analysis of the entire study sample. Although the inferior transfusion efficacy is one of the important concerns, the analysis of RPC-B transfusion for paediatric patients showed that a similar CCI was maintained between P-PC and RPC-B transfusions. ${ }^{15,20}$ Further investigation regarding the transfusion efficacy of RPC-B for adult patients is necessary. Furthermore, even the kind of PAS used for the washed PC products may be associated with the occurrence of ATRs. ${ }^{9}$ Therefore, the presently studied RPC-B transfusion may not be sufficient; further improvement may be necessary for the development of safe transfusion medicine.

However, this study has several limitations. Although we investigated medical records for a history of allergies, smoking, and drinking, we were not able to obtain this information for all patients. Therefore, some patients may have had an unknown allergy to foods or drugs, which could not be analysed in relation to the occurrence of ATRs, potentially causing bias. This is considered one of the inherent limitations of a retrospective analysis. Furthermore, various reports have been published about factors associated with the development of ATRs, and the results of those reports are not all in agreement with our results. Most likely, multiple factors, including race and differences in blood product preparation in each country, may add to the complexity of making such comparisons. Because this study was conducted at a single institution and thus targeted patients of the same race treated with PC products produced with consistent manufacturing methods, it may have been easier to draw some of these conclusions. However, we must examine whether the results obtained in this study could be generalized to other populations or regions. Further analyses of multiple populations will also be necessary. Furthermore, we did not analyse other factors, such as donor-related factors, productrelated factors (e.g., storage periods), ${ }^{33,34}$ or transfusion methods. ${ }^{35}$ Many factors may impact the occurrence of ATRs, and many things are still not understood.

In conclusion, this study shows that underlying disease and age are associated with the occurrence of ATRs resulting from PC transfusion. Furthermore, the onset of ATRs may be reduced when FFP products are used during the same period. Although haematological disease was one of the greater risk factors for developing ATRs, the provision of plasma-removed PC was effective in the prevention of ATRs in these patients. A safer transfusion therapy is possible if we can provide plasma-removed PC to more patients. However, it is impossible in the current situation to provide plasma-removed PC to all patients. Therefore, it is also important to adopt ATR precautions by determining ATR onset risk. Thus, safe and efficient transfusion medicine could be possible if we can provide plasma-removed PC to higher-risk patients preferentially. Further analysis of multiple aspects in prospective clinical trials with a larger number of patients is necessary to answer many questions. 


\section{References}

1. Kato H, Nakayama T, Uruma M, Okuyama Y, Handa M, Tomiyama Y, Shimodaira S, Takamoto S. A retrospective observational study to assess adverse transfusion reactions of patients with and without prior transfusion history. Vox Sang 2015;108: 243-50.

2. Oakley FD, Woods M, Arnold S, Young PP. Transfusion reactions in pediatric compared with adult patients: a look at rate, reaction type, and associated products. Transfusion 2015;55: 563-70.

3. Gauvin F, Lacroix J, Robillard P, Lapointe H, Hume H. Acute transfusion reactions in the pediatric intensive care unit. Transfusion 2006;46: 1899-908.

4. Savage WJ, Tobian AA, Savage JH, Wood RA, Schroeder JT, Ness PM. Scratching the surface of allergic transfusion reactions. Transfusion 2013;53: 1361-71.

5. Hirayama F. Current understanding of allergic transfusion reactions: incidence, pathogenesis, laboratory tests, prevention and treatment. Br J Haematol 2013;160: 434-44.

6. Tobian AA, Savage WJ, Tisch DJ, Thoman S, King KE, Ness PM. Prevention of allergic transfusion reactions to platelets and red blood cells through plasma reduction. Transfusion 2011;51: 1676-83.

7. Savage WJ, Savage JH, Tobian AA, Thoburn C, Hamilton RG, Schroeder JT, Ness PM. Allergic agonists in apheresis platelet products are associated with allergic transfusion reactions. Transfusion 2012;52: 575-81.

8. Savage WJ, Tobian AA, Fuller AK, Wood RA, King KE, Ness PM. Allergic transfusion reactions to platelets are associated more with recipient and donor factors than with product attributes. Transfusion 2011;51: 1716-22.

9. van Hout FMA, van der Meer PF, Wiersum-Osselton JC, Middelburg RA, Schipperus MR, van der Bom JG, Kerkhoffs JL. Transfusion reactions after transfusion of platelets stored in PAS-B, PAS-C, or plasma: a nationwide comparison. Transfusion 2018;58: 1021-7.

10. Kato H, Nakayama T, Uruma M, Okuyama Y, Handa M, Tomiyama Y, Shimodaira S, Takamoto S. Repeated exposure rather than the total volume of transfused components may influence the incidence of allergic transfusion reactions. Transfusion 2015;55: 2576-81.

11. Savage WJ, Hamilton RG, Tobian AA, Milne GL, Kaufman RM, Savage JH, Borge PD, Ness PM. Defining risk factors and presentations of allergic reactions to platelet transfusion. $\mathbf{J}$ Allergy Clin Immunol 2014;133: 1772-5 e9.

12. Kennedy LD, Case LD, Hurd DD, Cruz JM, Pomper GJ. A prospective, randomized, doubleblind controlled trial of acetaminophen and diphenhydramine pretransfusion medication versus placebo for the prevention of transfusion reactions. Transfusion 2008;48: 2285-91.

13. Marti-Carvajal AJ, Sola I, Gonzalez LE, Leon de Gonzalez G, Rodriguez-Malagon N. Pharmacological interventions for the prevention of allergic and febrile non-haemolytic 
transfusion reactions. Cochrane Database Syst Rev 2010: CD007539.

14. Tobian AA, King KE, Ness PM. Transfusion premedications: a growing practice not based on evidence. Transfusion 2007;47: 1089-96.

15. Kojima S, Yanagisawa R, Tanaka M, Nakazawa Y, Shimodaira S. Comparison of administration of platelet concentrates suspended in M-sol or BRS-A for pediatric patients. Transfusion 2018;58: 2952-8.

16. Oikawa S, Sasaki D, Kikuchi M, Sawamura Y, Itoh T. Comparative in vitro evaluation of apheresis platelets stored with $100 \%$ plasma versus bicarbonated Ringer's solution with less than 5\% plasma. Transfusion 2013;53: 655-60.

17. Oikawa S, Taguchi T, Endo K, Hoshi T, Kawashima W, Horibe Y, Urano S, Suzuki K, Minegishi M, Itoh T, Shimizu H. Storage of washed platelets in BRS-A platelet additive solutions based on two types of clinically available bicarbonated Ringer's solutions with different electrolyte concentrations. Transfus Apher Sci 2015;53: 233-7.

18. Fujiwara SI, Fujishima N, Kanamori H, Ito M, Sugimoto T, Saito S, Sakaguchi T, Nagai K, Masuoka H, Nagai K, Morita A, Kino S, Tanaka A, Hasegawa Y, Yokohama A, Fujino K, Makino S, Matsumoto M, Takeshita A, Muroi K. Released washed platelet concentrates are effective and safe in patients with a history of transfusion reactions. Transfus Apher Sci 2018;57: 746-51.

19. Ikebe E, Matsuoka S, Tanaka A, Yonemura Y, Fujii Y, Ohsaka A, Okazaki H, Kitazawa J, Ohtani S, Nakayama T, Momose SY, Miwa I, Taira R, Toyota K, Kino S, Kato H, Hamaguchi I. Reduction in adverse transfusion reactions with increased use of washed platelet concentrates in Japan-A retrospective multicenter study. Transfus Apher Sci 2019;58: 162-8.

20. Kobayashi J, Yanagisawa R, Ono T, Tatsuzawa Y, Tokutake Y, Kubota N, Hidaka E, Sakashita K, Kojima S, Shimodaira S, Nakamura T. Administration of platelet concentrates suspended in bicarbonated Ringer's solution in children who had platelet transfusion reactions. Vox Sang 2018;113: 128-35.

21. Yanagisawa R, Shimodaira S, Kojima S, Nakasone N, Ishikawa S, Momose K, Honda T, Yoshikawa K, Saito S, Tanaka M, Nakazawa Y, Sakashita K, Shiohara M, Akino M, Hirayama J, Azuma H, Koike K. Replaced platelet concentrates containing a new additive solution, M-sol: safety and efficacy for pediatric patients. Transfusion 2013;53: 2053-60.

22. Kanda Y. Investigation of the freely available easy-to-use software 'EZR' for medical statistics. Bone Marrow Transplant 2013;48: 452-8.

23. Kaufman RM, Assmann SF, Triulzi DJ, Strauss RG, Ness P, Granger S, Slichter SJ. Transfusion-related adverse events in the Platelet Dose study. Transfusion 2015;55: 144-53.

24. Osselaer JC, Cazenave JP, Lambermont M, Garraud O, Hidajat M, Barbolla L, Tardivel R, Defoin L, Waller C, Mendel I, Raidot JP, Kandel G, De Meuter R, Fabrigli P, Dehenau D, 
Arroyo JL, Padron F, Gouezec H, Corral M, Jacquet M, Sundin D, Lin L, Corash L. An active haemovigilance programme characterizing the safety profile of 7437 platelet transfusions prepared with amotosalen photochemical treatment. Vox Sang 2008;94: 315-23.

25. Yanagisawa R, Shimodaira S, Sakashita K, Hidaka Y, Kojima S, Nishijima F, Hidaka E, Shiohara M, Nakamura T. Factors related to allergic transfusion reactions and febrile nonhaemolytic transfusion reactions in children. Vox Sang 2016;110: 376-84.

26. Davoine F, Lacy P. Eosinophil cytokines, chemokines, and growth factors: emerging roles in immunity. Front Immunol 2014;5: 570.

27. Vossoughi S, Perez G, Whitaker BI, Fung MK, Stotler B. Analysis of pediatric adverse reactions to transfusions. Transfusion 2018;58: 60-9.

28. Slonim AD, Joseph JG, Turenne WM, Sharangpani A, Luban NL. Blood transfusions in children: a multi-institutional analysis of practices and complications. Transfusion 2008; $\mathbf{4 8}$ : 73-80.

29. Kato H, Uruma M, Okuyama Y, Fujita H, Handa M, Tomiyama Y, Shimodaira S, Kurata Y, Takamoto S. Incidence of transfusion-related adverse reactions per patient reflects the potential risk of transfusion therapy in Japan. Am J Clin Pathol 2013;140: 219-24.

30. Cognasse F, Payrat JM, Corash L, Osselaer JC, Garraud O. Platelet components associated with acute transfusion reactions: the role of platelet-derived soluble CD40 ligand. Blood 2008;112: 4779-80; author reply 80-1.

31. Heddle NM, Klama L, Singer J, Richards C, Fedak P, Walker I, Kelton JG. The role of the plasma from platelet concentrates in transfusion reactions. N Engl J Med 1994;331: 625-8.

32. Savage W, Tobian AA, Ness PM, Kaufman RM. Desensitization in allergic transfusion reactions: evidence from the Trial to Reduce Alloimmunization to Platelets. Transfusion 2014;54: 496-8.

33. Losos M, Biller E, Li J, Blower L, Hamad D, Patel G, Scrape S, Cataland S, Chen J. Prolonged platelet storage associated with increased frequency of transfusion-related adverse events. Vox Sang 2018;113: 170-6.

34. Kreuger AL, Caram-Deelder C, Jacobse J, Kerkhoffs JL, van der Bom JG, Middelburg RA. Effect of storage time of platelet products on clinical outcomes after transfusion: a systematic review and meta-analyses. Vox Sang 2017;112: 291-300.

35. Savage WJ, Tobian AA, Savage JH, Hamilton RG, Borge PD, Kaufman RM, Ness PM. Transfusion and component characteristics are not associated with allergic transfusion reactions to apheresis platelets. Transfusion 2015;55: 296-300. 
Table 1. Physical description of patients with or without alle rgic transfusion reactions

\begin{tabular}{|c|c|c|c|c|c|c|}
\hline Patient parameters & Patients with ATR $(n=65)$ & Patients without ATR $(\mathrm{n}=849)$ & P-value ${ }^{1)}$ & Risk ratio $^{2)}$ & $95 \% \mathrm{CI}^{2)}$ & P-value ${ }^{3)}$ \\
\hline Age (yrs); median (range) & $59(20-84)$ & $70(21-98)$ & $<0.001$ & 6.16 & $3.18-11.94$ & $<0.001$ \\
\hline Height (m); median (range) & $1.64(1.44-1.85)$ & $1.62(1.18-1.91)$ & 0.161 & 0.73 & $0.45-1.18$ & 0.236 \\
\hline Weight (kg); median (range) & $55.6(33.4-85.0)$ & $56.2(26.6-117.7)$ & 0.915 & 1.17 & $0.73-1.89$ & 0.602 \\
\hline $\mathrm{BSA}\left(\mathrm{m}^{2}\right) ;$ median (range) & $1.60(1.19-2.00)$ & $1.59(1.03-2.32)$ & 0.746 & 0.83 & $0.51-1.34$ & 0.511 \\
\hline BMI $\left(\mathrm{kg} / \mathrm{m}^{2}\right)$; median (range) & $20.9(15.4-28.3)$ & $21.6(12.0-45.0)$ & 0.325 & 1.30 & $0.80-2.11$ & 0.296 \\
\hline
\end{tabular}

ABBREVIATIONS: ATR(s), allergic transfusion reaction(s); BSA, body surface area; BMI, body mass index CI, confidence interval.

1) Statistical analysis was performed using Mann-Whitney $U$-test

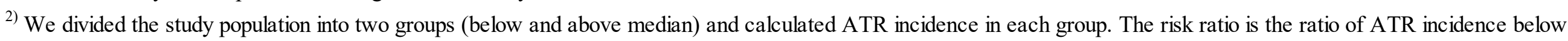
median and that above median.

${ }^{3)}$ Statistical analysis was performed using Fisher's exact test 
Table 2(A). Patient risk factors for the development of ATRs

(A) Univariate analysis

\begin{tabular}{|c|c|c|c|c|c|}
\hline \multirow{2}{*}{$\begin{array}{l}\text { Variable } \\
\text { Age }\end{array}$} & \multirow[t]{2}{*}{$\begin{array}{c}\text { Number of total } \\
\text { patients }(\mathrm{N}=914)\end{array}$} & \multicolumn{3}{|c|}{$\begin{array}{l}\text { Number of patients } \\
\text { with ATR }(\mathrm{N}=65)\end{array}$} & \multirow[t]{2}{*}{$\mathrm{P}$-value } \\
\hline & & & & & \\
\hline$\leq 40$ years & 66 & 15 & 22.7 & $\%$ & \\
\hline$>40, \leq 60$ years & 156 & 18 & 11.5 & $\%$ & $<0.001$ \\
\hline$>60$ years & 692 & 32 & 4.6 & $\%$ & \\
\hline \multicolumn{6}{|l|}{ Sex } \\
\hline Male & 566 & 38 & 6.7 & $\%$ & \multirow{2}{*}{0.597} \\
\hline Female & 348 & 27 & 7.8 & $\%$ & \\
\hline \multicolumn{6}{|l|}{ Allergic history ${ }^{1)}$} \\
\hline Food allergy & 75 & 11 & 14.7 & $\%$ & \multirow{2}{*}{0.021} \\
\hline None & 662 & 45 & 6.8 & $\%$ & \\
\hline Drug allergy & 227 & 25 & 11.0 & $\%$ & \multirow{2}{*}{0.025} \\
\hline None & 566 & 35 & 6.2 & $\%$ & \\
\hline \multicolumn{6}{|l|}{ Smoking history ${ }^{1)}$} \\
\hline None & 385 & 32 & 8.3 & $\%$ & \multirow{3}{*}{0.212} \\
\hline Quitting smoking & 78 & 2 & 2.6 & $\%$ & \\
\hline Smoking & 335 & 26 & 7.8 & $\%$ & \\
\hline \multicolumn{6}{|l|}{ Drinking history ${ }^{1)}$} \\
\hline None & 493 & 36 & 7.3 & $\%$ & \multirow{3}{*}{0.795} \\
\hline Quitting drinking & 228 & 18 & 7.9 & $\%$ & \\
\hline Drinking & 67 & 6 & 9.0 & $\%$ & \\
\hline \multicolumn{6}{|l|}{ Primary disease of patients } \\
\hline Hematological diseases & 138 & 46 & 33.3 & $\%$ & \multirow{4}{*}{$<0.001$} \\
\hline Emergency disease & 269 & 4 & 1.5 & $\%$ & \\
\hline Surgical disease & 245 & 4 & 1.6 & $\%$ & \\
\hline Other disease & 262 & 11 & 4.2 & $\%$ & \\
\hline \multicolumn{6}{|l|}{ ABO blood group } \\
\hline A & 346 & 27 & 7.8 & $\%$ & \multirow{4}{*}{0.387} \\
\hline $\mathrm{B}$ & 201 & 10 & 5.0 & $\%$ & \\
\hline $\mathrm{O}$ & 267 & 18 & 6.7 & $\%$ & \\
\hline $\mathrm{AB}$ & 100 & 10 & 10.0 & $\%$ & \\
\hline \multicolumn{6}{|c|}{ Number of times receiving PC products } \\
\hline$<5$ times & 746 & 49 & 6.6 & $\%$ & \multirow{3}{*}{0.337} \\
\hline $6-10$ times & 88 & 8 & 9.1 & $\%$ & \\
\hline$\geq 10$ times & 80 & 8 & 10.0 & $\%$ & \\
\hline \multicolumn{6}{|l|}{ Transfused period } \\
\hline$<7$ days & 694 & 42 & 6.1 & & \multirow{4}{*}{0.040} \\
\hline $7-14$ days & 32 & 3 & 9.4 & & \\
\hline $15-28$ days & 33 & 1 & 3.0 & $\%$ & \\
\hline$\geq 28$ days & 155 & 19 & 12.3 & $\%$ & \\
\hline \multicolumn{6}{|l|}{ Other transfused products } \\
\hline None & 125 & 21 & 16.8 & $\%$ & \multirow{4}{*}{$<0.001$} \\
\hline $\mathrm{RBC}$ & 185 & 35 & 18.9 & $\%$ & \\
\hline FFP & 24 & 0 & 0.0 & $\%$ & \\
\hline $\mathrm{RBC}$ and FFP & 580 & 9 & 1.6 & $\%$ & \\
\hline
\end{tabular}

(B) Multivariate analysis

\begin{tabular}{lll}
\hline Variables & Odds ratio $(95 \% \mathrm{CI})$ & P value \\
\hline Age & $0.470(0.313-0.706)$ & $<0.001$ \\
Primary disease of patients & $0.457(0.353-0.592)$ & $<0.001$ \\
Other transfused products & $0.429(0.315-0.584)$ & $<0.001$
\end{tabular}

Abbreviations: ATR, allergic transfusion reaction; CI, confidence interval; FFP, fresh frozen plasma; $\mathrm{PC}$, platelet concentrate; $\mathrm{RBC}$, red blood cell concentrate. 


\begin{tabular}{|c|c|c|c|c|c|c|c|}
\hline & \multicolumn{3}{|c|}{ P-PC } & \multicolumn{3}{|c|}{ RPC-B } & \multirow[b]{2}{*}{$\mathrm{P}$-value } \\
\hline & $\begin{array}{l}\text { Number of patients } \\
\text { with transfusion }\end{array}$ & Patients with ATR(s) & Ratio & $\begin{array}{l}\text { Number of patients with } \\
\text { transfusion }\end{array}$ & Patients with ATR(s) & Ratio & \\
\hline Total patients & 914 & 65 & $7.1 \%$ & 40 & 5 & $12.5 \%$ & 0.207 \\
\hline Hematological disease & 138 & 46 & $33.3 \%$ & 32 & 4 & $12.5 \%$ & 0.019 \\
\hline Other disease & 776 & 19 & $2.4 \%$ & 8 & 1 & $12.5 \%$ & 0.188 \\
\hline
\end{tabular}

\begin{tabular}{|c|c|c|c|c|c|c|c|}
\hline & & & & \multicolumn{3}{|c|}{ RPC-B } & \\
\hline & $\begin{array}{l}\text { Number of transfused } \\
\text { products }\end{array}$ & Products causing ATR(s) & Ratio & $\begin{array}{l}\text { Number of transfused } \\
\text { products }\end{array}$ & Products causing ATR(s) & Ratio & $\mathrm{P}$-value \\
\hline $\begin{array}{l}\text { Total patients } \\
\text { Only patients who underwent transition to RPC-B }{ }^{(1)}\end{array}$ & $\begin{array}{l}4677 \\
(247)\end{array}$ & $\begin{array}{c}92 \\
(23)\end{array}$ & $\begin{array}{l}2.0 \% \\
(9.3 \%)\end{array}$ & 490 & 6 & $1.2 \%$ & $\begin{array}{c}0.299 \\
(<0.001)\end{array}$ \\
\hline $\begin{array}{l}\text { Hematological disease } \\
\text { Only patients who underwent transition to RPC- } \mathrm{B}^{(1)}\end{array}$ & $\begin{array}{l}2047 \\
(247)\end{array}$ & $\begin{array}{l}70 \\
(23)\end{array}$ & $\begin{array}{l}3.4 \% \\
(9.3 \%)\end{array}$ & 453 & 5 & $1.1 \%$ & $\begin{array}{c}0.006 \\
(<0.001)\end{array}$ \\
\hline Other disease ${ }^{(2)}$ & 2630 & 22 & $0.8 \%$ & 37 & 1 & $2.7 \%$ & 0.276 \\
\hline
\end{tabular}

\begin{tabular}{|c|c|c|c|c|c|}
\hline \multicolumn{6}{|l|}{ (3) Transfusion response } \\
\hline & \multicolumn{2}{|r|}{ P-PC } & \multicolumn{2}{|c|}{ RPC-B } & \multirow[b]{2}{*}{ P-value } \\
\hline & $\begin{array}{c}\text { Evaluable number of } \\
\text { transfusions }\end{array}$ & $\begin{array}{c}\mathrm{CCI} 24 \mathrm{~h}\left(\times 10^{10} / \mathrm{L}\right. \\
\text { median } \pm \mathrm{SD})\end{array}$ & $\begin{array}{c}\text { Evaluable number of } \\
\text { transfusions }\end{array}$ & $\begin{array}{l}\mathrm{CCI} 24 \mathrm{~h}\left(\times 10^{10} / \mathrm{L} ;\right. \\
\text { median } \pm \mathrm{SD})\end{array}$ & \\
\hline Total patients & 213 & $1.56 \pm 0.70$ & 26 & $1.34 \pm 0.63$ & 0.022 \\
\hline Hematological disease & 101 & $1.41 \pm 0.57$ & 18 & $1.27 \pm 0.68$ & 0.218 \\
\hline Other disease & 112 & $1.73 \pm 0.75$ & 8 & $1.52 \pm 0.52$ & 0.109 \\
\hline
\end{tabular}

ABBREVIATIONS: ATR(s), allergic transfusion reaction(s); CCI 24h, post-transfusion corrected count increment after 24 hours; PC, platelet concentrate; P-PC, regular PC in plasma; RPC-B, resuspended PC in bicarbonate Ringer's solution and ACD-A (BRS-A) as plasma-replaced PC.

(1) Each value in parentheses represents limited patient's data who required transition to RPC-B products due to ATR with P-PC during the study period.

(2) No patient with another disease required transition to RPC-B products during the study period because of ATR due to P-PC. RPC-B products were transfused to these patients because of ATR that developed before the study period. 